\title{
INTERNACIONALIZACIÓN DE LA EDUCACIÓN SUPERIOR: CASO SUR DE PERÚ
}

\section{INTERNATIONALIZATION OF HIGHER EDUCATION: CASE SUR DE PERÚ}

\author{
${ }^{1}$ Silvia Cristina Quispe Prieto \\ ${ }^{2}$ Alberto Paucar Cáceres
}

\section{RESUMEN}

La Internacionalización de la Educación Superior representa una gran oportunidad de apertura al mundo, al fortalecimiento de la calidad educativa y a la contribución en la formación integral del educando universitario. Si asumimos que el rol de la internacionalización radica en que se trata de un "proceso de integración de una dimensión internacional/intercultural en las funciones de la enseñanza, la investigación y el servicio de la institución” (Knight 1994), se deriva que la concepción que asuma la universidad sobre la internacionalización va a contribuir directamente a implementar su misión y las acciones necesarias para implementar su estrategia.

La finalidad de este trabajo es múltiple: ofrecer aproximaciones para conceptualizar la Internacionalización en Educación Superior; explorar formas de cómo integrar la dimensión internacional en las funciones sustantivas de la universidad (la Internacionalización del currículo, Internacionalización de la investigación y posgrado e Internacionalización de las actividades de extensión universitaria y la cultura); e investigar cuáles son los elementos que debe impulsarse dentro de la gestión universitaria, con el fin de promover una cultura de internacionalización que sirva para desarrollar acciones estratégicas.

El proceso de internacionalización en las universidades peruanas se encuentra en diferentes niveles de desarrollo, cada una de manera aislada realiza esfuerzos por impulsar esta dimensión. Usando el marco teórico esbozado, se toma como referente a la Universidad Nacional Jorge Basadre Grohmann (UNJBG) de Tacna, a fin de describir sus experiencias en buenas prácticas de internacionalización durante estos últimos años. Concluimos que las universidades peruanas, entre ellas la UNJBG, vienen implementando estrategias de internacionalización. Sin embargo, para obtener el total beneficio de este proceso, se requiere de un trabajo articulado y coordinado, en el marco de una política de internacionalización de la educación superior en el Perú desde el Ministerio de Educación y una política institucional desde la gestión universitaria de cada institución.

Palabras Claves: Buenas prácticas, educación Superior, estrategias, internacionalización, universidades.

\section{SUMMARY}

The internationalization of higher education represents a great opportunity to open up to the world, to strengthen the quality of education and to contribute to an integral education of university students. If we assume that the role of internationalization lies in "integrating an international / intercultural dimension in the functions of teaching, research and service of the institution" (Knight, 1994), it follows that through internationalization the universities will 
contribute directly to the enrichment of its mission and the implementation of actions necessary to fulfil its strategy.

The purpose of this work is multiple. First, it aims to offer approaches to conceptualize internationalization in higher education. Second, it aims to explore ways to integrate the international dimension into the substantive functions of the university (the internationalization of the curriculum, internationalization of research and postgraduate studies, and the internationalization of external activities and culture). Finally, this work aims to investigate which are the elements that should be promoted within the university management in order to facilitate an internationalization culture that serves to develop strategic actions.

The process of Internationalization in Peruvian universities is at different levels of development, with each level held in isolation to make efforts in order to promote internationalization. Using the theoretical framework outlined, the National University Jorge Basadre Grohmann (UNJBG) is taken as a case, to describe its experiences in good practices of internationalization in recent years. We conclude that Peruvian universities, including UNJBG, have been implementing internationalization strategies. However, to reach the full benefits of internalization, an articulated and coordinated work is required; a work that is within the framework of a policy of internationalization of higher education in Peru at the level of the Ministry of Education and an institutional policy from the university management of each institution.

Keywords: Good practices, higher, internationalization, strategies, universities education.

\section{INTRODUCCIÓN}

La internacionalización lleva implícita en su concepto las raíces de la globalización iniciada desde hace varios años; si bien es cierto, esta ha sido relacionada principalmente al aspecto económico, fue abarcando también a todas las instancias culturales, políticas e inclusive a la educación. Probablemente, este acelerado crecimiento se deba tanto a las redes de comunicación - las cuales permiten compartir experiencias desde países que pueden llevar un paso adelante y ofrecen posibilidades de adaptación a nuestra realidad - como a la inherente capacidad de las universidades para interactuar en procesos académicos, de investigación y extensión que han conllevado a incorporar una dimensión internacional e intercultural, dependiendo de la concepción asumida por la institución superior.

La internacionalización representa una apertura al mundo, el fortalecimiento de la calidad educativa y la indiscutible contribución en la formación integral del educando universitario; asimismo, implica nuevas oportunidades que se iniciaron en programas de movilidad cuyos principales actores son docentes-investigadores $\mathrm{y}$ estudiantes, y que con el tiempo se fue estableciendo como eje trasversal de las principales funciones de las universidades: docencia, extensión e investigación.

Uno de los principales retos que debe enfrentar el Estado peruano es establecer políticas de internacionalización, es decir, institucionalizar dicho proceso con la intención de hacer frente a los obstáculos de la burocracia, la falta de financiamiento, la lentitud de los cambios estructurales para afrontar la globalización y el intercambio de conocimiento. Por su parte, las universidades vienen implementando estrategias y sumando esfuerzos para el logro de los objetivos de internacionalización que promuevan sus instituciones, en la búsqueda de insertarlas al contexto internacional y convertirlas en un destino importante dentro del ámbito de la educación superior. Para ello se debe fortalecer, además, capacidades de internacionalización y establecer alianzas estratégicas. 
Mientras no exista una política o estrategia formal asumida en las instituciones superiores, la tarea de internacionalización demandará mayores esfuerzos. A pesar de los obstáculos, durante los últimos años en las universidades públicas del interior se ha tratado de avanzar en este tema y aunque aún resta mucho camino que recorrer, se debe reconocer que una muy buena alternativa para su desarrollo ha sido el establecer estrategias interinstitucionales como, por ejemplo, ser partícipes de proyectos financiados por fondos europeos.

\section{DESARROLLO TEMÁTICO}

\section{Definición y significado de la Internacionalización de la Educación Superior (IES)}

Entre los autores más reconocidos que han aportado en la definición de la Internacionalización de la Educación Superior (IES), se encuentran Knight (1994) y Wit (1995) — citados por Pascual (2016) — quienes conciben a la IES como "el proceso de integración de una dimensión internacional/intercultural en las funciones de la enseñanza, la investigación y el servicio de la institución”(Knight, 1994).(Pascual, 2016).

Jane Knight, estudiosa en esta línea, realiza el análisis conceptual de la IES a través de la identificación de sus elementos constitutivos más esenciales al interior de las instituciones de educación superior canadienses, los cuales fueron propuestos en su libro Internationalization: elements and checkpoints, editado por el Canadian Bureau for International Education, con la finalidad de ser útiles para la implementación y formalización de la internacionalización al interior de las universidades en Canadá (Hernández, 2016).

Según Knight (1994), la internacionalización ha sido entendida como un enfoque organizacional que considera la construcción de una cultura de la internacionalización, a partir de una perspectiva de logro de competencias en la formación de los estudiantes; así también, como una perspectiva de actividades internacionales referidas a la implementación de las mismas en el desarrollo curricular, movilidad y cooperación académica; o como un proceso en el cual se incorpora una dimensión internacional en las funciones sustantivas de la institución. Knight se inclina por esta última definición, conceptualizando la Internacionalización de la Educación Superior dentro de las funciones de la universidad. Al respecto, Hernández (2016) señala:

Esta nueva definición de la Internacionalización de la educación va mucho más allá de la definición de internacionalización como la sumatoria de actividades y programas dada por otros autores [...] y lleva la internacionalización hacia una concepción más integral.

Desde el enfoque integral del concepto de la IES, Knight propone once elementos que debe considerarse para implementar y fortalecer la internacionalización a nivel institucional:

- Apoyo administrativo y organizacional.

- Estudiantes Internacionales.

- Estudiantes locales y su participación en actividades internacionales.

- Currículo.

- Investigación.

- Desarrollo Internacional y Asistencia Técnica.

- Aprendizaje de una lengua extranjera.

- Formación y convenio educativos.

- Cooperación y alianzas externas.

- Acuerdos y Vínculos académicos internacionales.

- Servicios Institucionales y Actividades Extracurriculares.

Para la operacionalización de los elementos propuestos por Knight, se requiere un cambio de paradigma en la comunidad universitaria a fin de ir evolucionando e integrando con mayor profundidad cada elemento. La autora señalada refiere que se trata de un proceso necesario para institucionalizar y fortalecer la gestión de la internacionalización dentro de la gestión universitaria; señala además que dicho proceso debe iniciarse con la Cultura de respaldo a la internacionalización que cada institución tiene 
de manera elemental, los miembros integrantes de la comunidad universitaria deben conocer y estar convencidos tanto de la importancia como de los beneficios de la internacionalización de la educación. El ciclo se desarrollaría de la siguiente manera:

- Conciencia: Identificar las bondades e importancia de la internacionalización.

- Compromiso: Implica el respaldo y voluntad política de las autoridades.

- Planeación: Establecer prioridades, acciones y estrategias.

- Operacionalización: Implementación de las actividades y servicios administrativos necesarios para la gestión de la estrategia.

- Revisión: Referido a la evaluación de resultados institucionales que permitirá la sostenibilidad y perfeccionamiento a mediano y largo plazo.

- Fortalecimiento: Motivar e involucrar la participación de la comunidad universitaria y continuidad e impulso en la tarea de la internacionalización. (Hernández, 2016)

\section{Por dónde empezar: Visibilizando indicadores para el Diagnóstico de la IES}

Existen esfuerzos aislados de las universidades por impulsar la internacionalización en sus instituciones. Basados en el significado de la IES y los conceptos que involucra, explicados anteriormente, se plantea partir de la valoración previa de esta dimensión institucional, así como conocer su realidad; esto debido a que uno de los principios básicos de la gestión de la calidad es el enfoque objetivo que conduce a la toma de decisiones, o lo que es lo mismo: las decisiones deben basarse en el análisis de datos y de la información disponible. Por consiguiente, para realizar este proceso de manera eficaz y eficiente se debe disponer de datos medidos de forma sistemática, periódica y precisa. La forma de hacerlo es a través del establecimiento de indicadores que permitan partir de una línea base, es decir, un diagnóstico que denote el nivel de IES, los elementos que han sido implementados y cuáles no se encuentran o requieren ser reforzados (Vicerrectoría, 2014).
La Comisión Interinstitucional de Internacionalización del Consorcio de Universidades Mexicanas (CUMex), realizada en el año 2011, plantea un manual de indicadores de Internacionalización, para lo cual considera la integración de la dimensión internacional en las funciones sustantivas de las universidades; por tanto, se debe diseñar la estructura programática desde los siguientes aspectos propuestos:

$>$ Internacionalización del currículo. Está considerada como la "acción estratégica que implica conjunto de acciones, principios y objetivos que impactan en la totalidad de la vida del campus universitario" (Vicerrectoría, 2014). Esto involucra promover, organizar y sostener programas de movilidad física y virtual de docentes y estudiantes; integrar una perspectiva internacional, intercultural, global y comparativa; organizar programas académicos conjuntos internacionales; considerar programas de enseñanza de idiomas y culturas extranjeras; así como incorporar profesores invitados en los cursos regulares; fomentar la experiencia internacional del personal académico; contar con la presencia de estudiantes extranjeros, y todo aquello que permita la incorporación de la institución a las tendencias mundiales en materia de currículo.

> Internacionalización de la Investigación y posgrado. Organizar programas de movilidad de investigadores; promover y apoyar proyectos de investigación con participación de investigadores extranjeros; apoyar proyectos de cooperación para el desarrollo; organizar posgrados de excelencia en colaboración con instituciones internacionales; fomentar los programas en cotutela con instituciones extranjeras y las tutorías de tesis por parte de académicos de instituciones internacionales; así mismo CUMex propone alentar la participación de redes internacionales de investigación, ofertas transnacionales de programas de posgrado e incorporación de alumnos extranjeros.

Internacional de la extensión y la cultura.Corresponde alentar a la membresía institucional en redes e instituciones internacionales promotoras de la cooperación en materia de Educación Superior; organizar 
eventos internacionales de difusión cultural, cátedras internacionales y difusión internacional. (Consorcio, 2011)

\section{Estrategias en la Internacionalización}

Es importante no olvidar el ciclo de implementación de la Cultura de internacionalización para impulsar las estrategias de cambio institucional hacia un nivel mayor en las universidades, que conlleven a la transformación de la institución a través de la IES integrándola a la misión, metas, organización y programas con estrategias organizacionales y programáticas. "Las primeras referidas a implementar políticas institucionales administrativas y organizacionales y las segundas hacen referencia a la puesta en marcha de programas y actividades para internacionalizar la docencia, investigación y extensión" (Vicerrectoría, 2014), (Knight, 1994).

Las estrategias programáticas consisten en la implementación de programas académicos, mediante los cuales se desarrolla la internacionalización de las funciones sustantivas de la universidad. Por otro lado, las estrategias organizacionales tienen como objetivo la institucionalización de la dimensión internacional en las políticas generales, misión, procedimientos y reglamentos administrativos de la institución. Según CUMex, las estrategias programáticas responden a la pregunta ¿qué se tiene que integrar en la IES?; mientras la función de las estrategias organizacionales es ocuparse de cómo hacerlo.

Dentro de las estrategias organizacionales, se menciona en Consorcio (2011) la integración de la dimensión de la internacionalización en los siguientes aspectos institucionales:

- Políticas en materia de docencia, investigación y extensión.

- Planeación institucional.

- La presupuestación.

- Aseguramiento de la calidad y gestión.

- Normatividad de la institución.

- Organización de una oficina con suficiente rango jerárquico y autonomía, pues deberá coordinar y gestionar todo el proceso de internacionalización institucional, así como las actividades correspondientes.

En relación a las estrategias programáticas, Consorcio (2011) consideró lo siguiente:

- Convenios de colaboración.

- Experiencia internacional de los estudiantes a través de movilidad.

- El perfil internacional de los recursos humanos para la docencia e investigación.

- Participación en redes institucionales de investigación y docencia.

- Integración internacional en programas de estudios y en las disciplinas.

- Establecimientos de programación académica en colaboración internacional (ej. Máster compartidos).

- Fomentar el aprendizaje de idiomas y culturas extranjeras.

- Presencia de estudiantes extranjeros.

- Modalidades de educación trasnacional, eventos académicos conjuntos.

- Movilidad virtual.

- Proyectos de investigación en cooperación y colaboración con instituciones o asociaciones.

- Participación en organismos y asociaciones internacionales.

\section{Internacionalización de las Universidades: Caso Sur del Perú}

Durante los últimos años se ha viabilizado un compromiso asumido por las universidades y las autoridades ministeriales de los países involucrados, a fin de incorporar la internacionalización tanto en la gestión universitaria como en sus funciones esenciales; por cuanto en América latina, la IES fue incrementándose. En el caso específico de Perú, la Ley Universitaria marcó un punto de inflexión en el sistema universitario, con un modelo de la calidad que exige adaptarse a nuevos estándares y mantener un proceso de mejora continua en las universidades peruanas, en razón a ello, muchas de ellas ya se han sometido a mecanismos de acreditación por parte de organismos internacionales. 
En octubre de 2018, la revista América Economía publicó el ranking de las mejores universidades de Perú en su reportaje de educación, ubicando en la dimensión internacionalización a instituciones privadas entre las 10 mejores del país; para ello consideró entre sus indicadores de evaluación, la calidad de las universidades extranjeras con las cuales se vincularon para el intercambio de profesores y estudiantes. (Riquelme, 2018). Si bien es cierto son pocas las universidades públicas que figuran en los primeros lugares de desarrollo de la internacionalización, no puede negarse que la mayoría de ellas muestra una preocupación latente por lograr la ansiada calidad de la educación superior; lo cual conlleva a mayores exigencias de un personal cada vez más calificado, con capacidad para la innovación y la adaptación, que demanda implementar la internacionalización con un enfoque sistémico y organizado a fin de formar ciudadanos globales.

Las instituciones involucradas en la Internacionalización de la Educación Superior en el Perú han desarrollado este proceso, a diferentes niveles; sus avances se dieron "principalmente por las iniciativas individuales y descoordinadas de algunas universidades" (British Council, 2017), las mismas que parten del concepto de la IES, así como a través de las estrategias que han implementado para d e sarrollar dicho proceso de internacionalización. Entre las acciones estratégicas que se visibilizan con un mayor avance, se encuentra la promoción del manejo de movilidad; participación en redes; proyectos conjuntos de investigación; impulso de aprendizaje del idioma inglés; difusión de oportunidades y promoción de visibilidad universitaria; internacionalización del currículo y /o doble titulación; participación en congresos, eventos académicos internacionales $\mathrm{y}$ en cursos cortos.

La acción estratégica de la movilidad promocionada a través de redes internacionales y nacionales, en mayor y menor grado, es lo que mayormente se ha implementado en las universidades del interior del país; por tanto, también es preciso tomar en cuenta el fortalecimiento de competencias de los recursos humanos para la gestión de la misma y su implementación a través de las oficinas de relaciones internacionales.

La Universidad Nacional Jorge Basadre Grohmann de Tacna - como universidad pública ubicada en el sur de Perú, zona costera frontera con Chile - pretende, a través de la internacionalización, favorecer el desarrollo sociocultural tanto como el acceso a un mayor conocimiento; así también busca mejorar la comunicación entre las universidades del país y del extranjero mediante la generación de alianzas estratégicas. Hace más de 10 años inició acciones estratégicas de internacionalización $\mathrm{y}$ ha concentrado gran parte de sus actividades en la movilidad estudiantil y docente, impulsando la investigación; sin embargo, el nivel de desarrollo de la internacionalización aún presenta muchos retos por alcanzar, situación similar a otras universidades del sur del país. Las oportunidades presentadas a través de alianzas estratégicas son las que, reportando un efecto multiplicador, han permitido mayor dinamismo entre universidades nacionales y extranjeras.

La UNJBG viene desarrollando y sumando esfuerzos con la finalidad de impulsar el trabajo de internacionalización. Esto se ha visto fortalecido a través de la formación e integración de redes nacionales e internacionales. Con la participación activa de la Oficina de Cooperación Nacional e Internacional de la UNJBG se impulsó la conformación de dos redes: REDISUR (Red de Integración de Universidades del Sur del Perú) y RIFU (Red de Integración Fronteriza Universitaria Perú-Chile); por medio de ellas se están realizando acciones con el objetivo de generar espacios de movilidad y vinculación para la investigación. Así también, forma parte de RPU (Red Peruana de Universidades) que agrupa a 21 instituciones, buscando promover la integración, el intercambio y la construcción de conocimiento entre ellas. Por otro lado, cabe resaltar que la UNJBG también es miembro de redes internacionales como CRISCOS (Consejo de Rectores por la Integración de la Subregión Centro oeste de Sudamérica) que reúne universidades de Argentina, Bolivia, 
Chile, Paraguay, Ecuador y Perú; cuyo mayor peso de trabajo recae en el intercambio estudiantil. Además, es partícipe de becas para docentes, estudiantes e investigadores ofertadas por la Alianza Pacífico - conformada por universidades de México, Colombia, Chile, Perú-. A nivel Internacional, en Europa es integrante del Grupo de universidades La Rábida.

Otra de las alianzas que representó un gran aporte para la internacionalización en la UNJBG fue EUROINKA, Proyecto de Cooperación Internacional auspiciado por el Programa Erasmus Mundus Action 2, que en un periodo de tres años vinculó a 20 prestigiosas instituciones de educación superior en Europa y América Latina. En dicho proyecto se efectuó un total de 182 intercambios académicos que incluyeron licenciatura, maestría, doctorado y post-doctorado donde los participantes recibieron el apoyo de la Comisión Europea a partir de subvenciones y becas otorgadas que, a excepción de tasas de matrícula, cubrieron sus gastos de viaje, seguro internacional y una asignación mensual de acuerdo con el nivel de formación. Este proyecto concluyó en julio de 2018.

En el caso específico de la UNJBG se realizó 16 movilidades entre estudiantes, docentes e investigadores; de los cuales, 8 han sido entrantes y 8 salientes. En cuanto a los estudiantes, el proyecto representó una gran oportunidad en su formación profesional. Un grupo fue movilizado a Europa (Universidad de Vigo y Universidad de Upssala); asimismo, se recibió a una estudiante de la Universidad de Manchester, quien cursó un semestre de estudios y realizó una investigación en el Amazonas cuyo informe fue publicado en un capítulo del libro University Initiatives in Climate Change Mitigation and Adaptation. Por otra parte, la Movilidad de Staff Académicos significó un valioso aporte en la dimensión de Internacionalización de la investigación, al permitir la llegada de 3 académicos investigadores de la Universidad Pablo de Olavide y de la Universidad de Vigo (ambas de España); quienes se integraron a proyectos de investigación de la UNJBG y que, en su mayoría, han continuado trabajando en problemáticas actuales y líneas prioritarias de la región de Tacna como son Variedades de cultivos tolerantes a boro en el departamento de Tacna, Impacto de la herbívora y del cambio climático en las zonas áridas, y Acuicultura del sargo o chita (Anisotremus scapularis) en el Perú. Entre estas buenas prácticas de internacionalización fueron presentados trabajos de investigación conjunta a Fondos Europeos, así como al Fondo de canon, sobre canon y regalías mineras, con resultados positivos. Otro gran aporte de este proyecto es la vinculación que se logró con las universidades socias, la cual brinda mejores oportunidades para el desarrollo de futuros proyectos.

Así como EUROINKA existen otras experiencias de proyectos financiados con el Fondo Europeo y que han permitido asociarse a universidades geográficamente ubicadas en el interior del Perú, como la Universidad Tecnológica de los Andes y la Universidad Nacional de Cajamarca, participantes del Proyecto PONCHO "Internacionalización de Universidades periféricas de América Latina a través de una integración sostenible y de una implementación inclusiva de Oficinas de Relaciones Internacionales", el cual tiene relación directa con la gestión de la internacionalización y fomento de intercambio de buenas prácticas de los socios más experimentados a los no tan experimentados. Esta experiencia fortalece sus competencias en las dimensiones de Formación, Internacionalización de la investigación y del Currículo. Debido a las características de participación en este proyecto, únicamente 2 universidades peruanas fueron incluidas.

Pese a la importante ayuda de la Comisión Europea, la demanda de mayores oportunidades de inclusión continúa creciendo. Frente a las necesidades de las universidades socias de América latina, aún queda mucho trabajo por hacer a fin de que se fortalezcan las capacidades humanas de sus miembros, así como sus capacidades estratégicas y de infraestructura.

Las experiencias de otras universidades que tienen mayor nivel de internacionalización son valiosas, nos dejan el desafío de lograr una mayor presencia académica y cultural. Por otro lado, también es importante que las 
instituciones universitarias propicien condiciones necesarias para generar un entorno favorable a la internacionalización como el nivel de inglés de los alumnos y docentes, asimismo se debe simplificar la complejidad de los procesos administrativos de reconocimiento de notas y títulos, y mejorar el sistema de acreditación (British Council, 2017).

Al respecto, Pedro Henríquez-Guajardo (2018), director del Instituto Internacional para la Educación Superior en América Latina y el Caribe (IESALC UNESCO) señaló lo siguiente:

Es necesario un mayor compromiso para el establecimiento de políticas públicas a nivel nacional y regional que fomenten el proceso de la internacionalización de las instituciones de educación superior. Además, se debe fortalecer las instancias de diálogo existentes para articular y darle seguimiento a las iniciativas de hermanamiento interinstitucional de las IES.

\section{CONCLUSIONES}

Entre las concepciones y significado de la IES, con un enfoque integral desde la perspectiva institucional, destacan los estudios académicos de Jane Knight. A fin de implementar la internacionalización, ella propone una serie de elementos que facilitan la operativización y el establecimiento de parámetros de avance en el interior de las universidades, resaltando además la cultura como respaldo para lograr la ansiada internacionalización. Esto implica que los miembros de la comunidad universitaria se concienticen sobre la importancia y bondades de la Internacionalización en su ciclo de Compromiso, Planeación, Operacionalización, Revisión y Fortalecimiento. Por consiguiente, será de gran ayuda establecer la línea base en donde se encuentra el proceso de IES, cuidando que este responda a los indicadores relacionados con las funciones sustantivas de la universidad.

Existen experiencias de buenas prácticas de internacionalización en las universidades del sur peruano; no obstante, de manera comparativa, unas difieren de otras en cuanto a niveles del proceso de internacionalización se refiere, entre las que sobresalen las universidades privadas.

Es importante, asimismo, desarrollar de manera coordinada y articulada políticas de IES desde el Ministerio de Educación y desde la gestión de la institución universitaria; propiciando para ello, entornos apropiados.

\section{REFERENCIAS BIBLIOGRÁFICAS}

British Council. (2017). La Reforma del Sistema Universitario Peruano: Internacionalización, Avance, Retos y Oportunidades. Recuperado el 4 de octubre de 2018, de British Council $\mathrm{P}$ e r ú https://www.britishcouncil.pe/sites/de fault/files/la_reforma_del_sistema_u niversitario-peruanointernacionalización avance retos $\overline{\mathrm{y}}$ _oportunidades.pdf

Consorcio, U. M. (2011). Manual de Indicadores de Internacionalización de la Educación Superior. México: CUMex.

Henríquez-Guajardo. (17 de agosto de 2018). Internacionalización de la Educación Superior. (?, Entrevistador).

Hernández, J. (2016). Comienzos de Jane Knight. La Internacionalización de la educación superior desde el contexto de la gestiòn institucional. España: El Glocalblog.

$\left.\mathrm{Kn} \mathrm{ig} \mathrm{h} \mathrm{t,} \mathrm{J} \mathrm{.} \mathrm{(} \mathrm{(} \begin{array}{llll}1 & 9 & 9 & 4\end{array}\right)$ Internationalization:Elements and Checkpoints. Ottawa: Canadian Bureau for International Education. Issue $\mathrm{N}^{0} 7$

Pascual, M. (2016). ¿De qué hablamos cuando hablamos de internacionalización universitaria argentina en los documentos oficiales? Aportes desde el análisis del discurso. Revista de Educación, Ciencia y Cultura. v 21 n.2, pp. 125-149.

Riquelme, S. C. (24 de octubre de 2018). Conozca el ránking de las mejores Universidades del Perú 2018. Recuperado el 26 de octubre de 2018, de Revista América Economía: https://mba.americaeconomia.com/ articulos/reportajes/conozca-elranking-d e-Ias-mejoresuniversidades-del-peru-2018

Vicerrectoría, I. (2014). Manual de Indicadores de Internacionalización. Honduras: UNAH. 